\title{
Secundarismos metastásicos peneanos. Sucinta revisión de la literatura urológica española (periodo 1980-2005)
}

\author{
Pellicé i Vilalta C. \\ Clínica de Nuestra Señora del Remedio. Barcelona.
}

Actas Urol Esp. 2006;30(9):962-964

\section{Sr. Director:}

En referencia a la Nota Clinica "Adenocarcinoma de próstata metastático en pene" aportada por Cortés y cols. en la revista Actas Urológicas Españolas (2006; 30:832), debo manifestarle que la comparto en su totalidad. Es de correcto redactado y es también convincente la iconografia que insertan (Macroscópica y microscópica). Los autores con sumo acierto, compendian lo fundamental a conocer acerca de tal atípica entidad, pero al revisar la bibliografia se aprecia la falta total y absoluta de citas de autores españoles que en lengua castellana ya han tratado la entidad. Los autores sólo aportan una $^{2}$ y ésta viene referenciada en lengua inglesa, cuando fue publicada en lengua castellana.

Recientemente he realizado una nueva revisión bibliográfica sobre el tema (Tabla 1).

De ésta se desprende que presumiblemente la primera cita en lengua castellana y aportada por autor español en revista urológica española sea la de Alonso y cols. de $1980^{2}$. Honradamente creo que este original es de obligada lectura al tratar estas temáticas. Han pasado ya 26 años y mantiene una total vigencia (Aspectos clinicos, diagnósticos, terapéuticos y pronósticos).

\section{Tabla 1}

Tumores metastásicos peneanos

\section{CPIV: Referencias bibliográficas personales} 1.- PUBLICACIÓN:

Annals d'Urologia 2006;6:1-13 (Web Societat Catalana d'Urologia - SCU) (*)

\section{2.- COMUNICACIONES:}

LI Congreso Nacional de la AEU: Pamplona; 1986

XXIII Reunión del Grupo Uro-Oncológico de la AEU: Granada; 2006

XIV Jornada de l' ACUC-A.: Cambrils; 2006

XII Simposi Institucional de la SCU: Girona; 2006

(*) Nota: Las tablas adjuntas (2-9) son tomadas y adaptadas de este trabajo, previa traducción de la lengua catalana a la lengua castellana realizada por el propio autor (CPiV).
Tabla 2

Tumores metastáticos peneanos

Referencias bibliográficas urológicas y de autores del estado español (período: 1980-2005)

Artículos publicados en Actas Urológicas Españolas ... . . .21

Artículos publicados en Archivos Españoles de Urología . . . . .6

Otras publicaciones $\ldots \ldots \ldots \ldots \ldots \ldots \ldots \ldots \ldots \ldots$

Abstracts congresos estado español . . . . . . . . . . ...9

TOTAL: 38 citas aportadas / 60 casos descritos

Tabla 3

Tumores metastáticos peneanos

Casuística estado español. Instituciones sanitarias (periodo: 1980 - 2005)

Fundación Puigvert (Barcelona): 1986-2004 ........................8 Casos

Hospital La Fe (Valencia): 1980-1986 ..................................8 Casos

Hospital Universitario (Murcia): 2000-2002 ….....................6 Casos

Hospital de Guipúzcoa: 1985-1999.......................................5 Casos

Hospital Clínico (Barcelona): 1986-1988................................5 Casos

Hospital Ramón y Cajal (Madrid): 1987-2004 .......................3 Casos

Hospital Clínico (Madrid): 1985-1991 .................................... Casos

Hospital Carlos Haya (Malaga): 1993 …………....................3 Casos

Hospital Clínico (Salamanca): 1982 .......................................1 Caso

Hospital de Bellvitge (L'Hospitalet del Ll.): 1984 ...................1 Caso

Fundación Jiménez Díaz (Madrid): 1990 .................................. 1 Caso

Hospital de Denia: 1991.....................................................1 Caso

Hospital de Estella: 1994 .................................................. Caso

Hospital de Sabadell: 1999-2000............................................ Caso

Hospital Virgen de la Arrixaca (Murcia): 2000 ........................1 Caso

Hospital N.S. De Sónsoles (Ávila): 2002 .................................1 Caso

Hospital de Miranda De Ebro: 2003 ....................................... Caso

Hospital de Calatayud: 2004 ...............................................1 Caso

Hospital de Getafe: 2005.....................................................1 Caso

Otras Instituciones ............................................................. Casos

TOTAL: 60 casos descritos / 38 citas aportadas 
Las casuísticas españolas más numerosas corresponden a la de la Fundació Puigvert de Barcelona y la del Hospital La Fe de Valencia, ambas con ocho casos.

Para no excederme en el redactado y bajo el formato de varias tablas, le aporto y resumo lo que ha sido del interés demostrado por autores españoles y en la literatura urológica española acerca de los secundarimos metastáticos peneanos (Tablas 2-9).

Pláceme remitirle una sucinta revisión de citas que hacen la referencia a las metástasis peneanas de tumores prostáticos.

\section{Tabla 4}

Tumores metastáticos peneanos

Casuística Estado Español (1980 - 2005)

Localización del tumor primario

(urogenital: 18/extra-urogenital: 12)

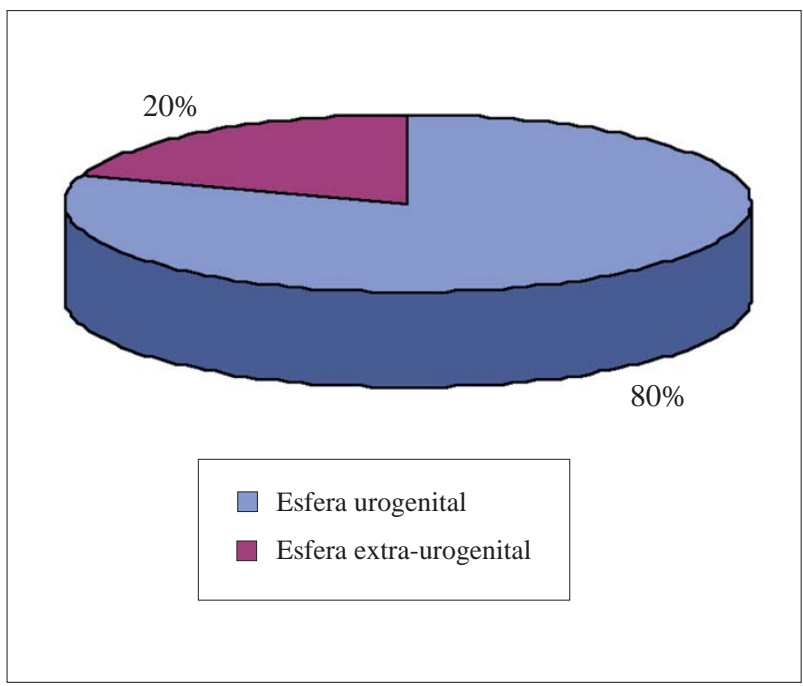

Tabla 5

Tumores metastáticos peneanos

Casuística estado español (1980 - 2005)

Localización del tumor primario

(48 casos: esfera urogenital)

Tumores uroteliales de vejiga $\ldots 24$

Adenocarcinomas prostáticos 15

Tumores renales tipo "hipernefroma" .................................5

Tumores de vías ...2

Tumor de testículo $\ldots 1$

Tumor prostático transcisional
Tabla 6

Tumores metastáticos peneanos

\section{Casuística estado español (1980 - 2005) Localización del tumor primario (12 casos: esfera extra-urogenital)}

Tumores de recto-sigma.......................................

Tumores de pulmón...................................................2

Tumor de esófago.............................................. 1

Sarcoma de Éwing ..................................................... 1

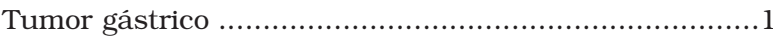

Tabla 7 (A y B)

Tumores metastáticos peneanos

Casuística estado español (1980 - 2005)

Formas de presentación clínica peneana local (frecuentes asociaciones entre ellas)

Efecto masa .29 casos

(prepucio /surcos /glande /cuerpos cavernosos)

únicos ..............19 casos
múltiples ............10 casos

Priapismo tumoral o maligno 19 casos

Luts y/o rao 15 casos

Cuadros álgicos importantes 14 casos

Ulceras y/o lesiones dermatológicas varias ........9 casos Grandes edemas 5 casos

Cuadros gangrenosos....................................4 casos

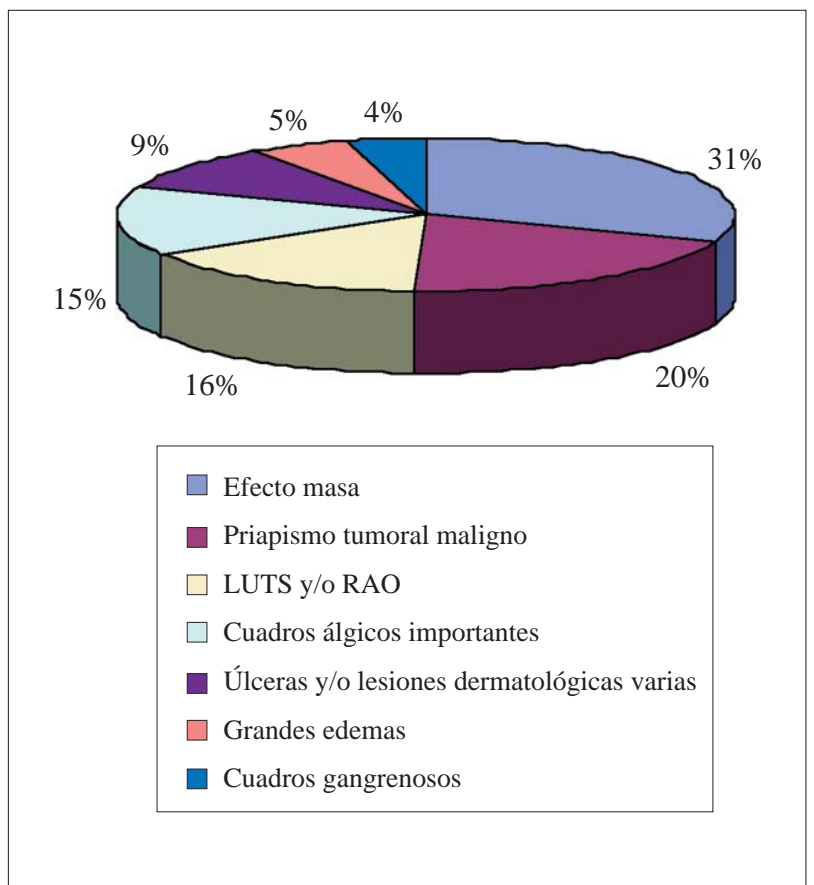


Tabla 8 (A, B y C)

Tumores metastáticos peneanos

\section{Casuística estado español (1980 - 2005) Priapismo tumoral maligno (19 casos)}

Tumores uroteliales de vejiga...........................6 casos Adenocarcinomas prostáticos ........................4 casos Tumores renales tipo "hipernefroma" ................4 casos Tumores de recto-sigma ................................... casos Tumor testicular ............................................. caso Tumor de vías ............................................ caso

Casuística estado español (1980 - 2005) Priapismo tumoral maligno (19 casos)

Tumor primario urogenital. 16 casos Tumor primario extra-urogenital...................... 3 casos

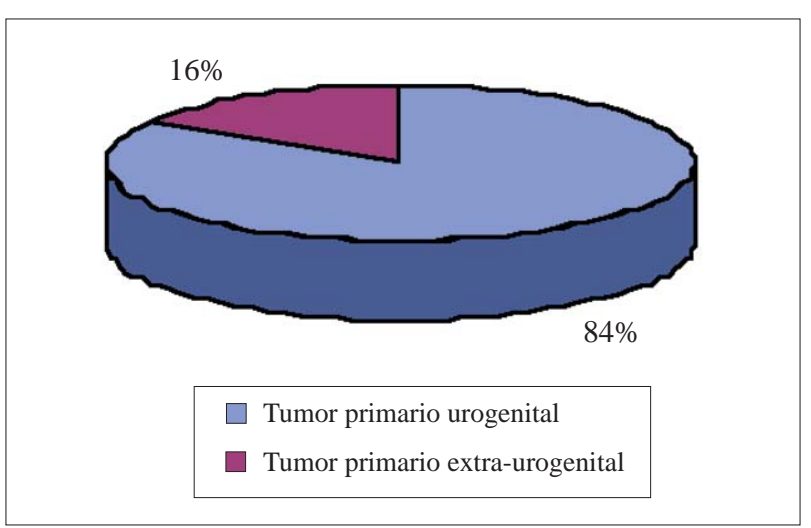

\section{REFERENCIAS}

1. Abad C, Prats J.; Garcia D, Cabezuelo M, Prera A, Vicente E, Bella M. Metástasis infrecuentes de tumores urológicos. Simposi Institucional de la Societat Catalana D'urologia (Terrassa; 2000) (Abstract).

2. Alonso M, Guillen M, Tramoyeres A, Rodríguez J, Ferrutxe J, Puig R. Tumores metastásicos de pene. Arch Esp Urol. 1980;4:131-142.

3. Bañon V, Nicolas J, Valdelvira P, Server G, Martinez E, Gomez G, Prieto A, Lopez P, Perez M. Neoplasias malignas del pene. Actas Urol Esp. 2000;24:652-658.

4. Garcia F, Garcia J, Tomas M, Rico J, Sempere A, Morga J, Fontana L. Infiltración neoplásica del pene secundaria a tumores de origen diverso. Nuestra experiencia. Congreso Nacional de Urología (AEU; Murcia 2002), Abstract: P.185.

5. Garcia D, Prats J, Prera A, Abad C, Bella M, Nogue M, Cabezuelo M, Luelmo J. Metástasis peneana de carcinoma transicional prostático. Jornada de L'associacio Catalana D'urolegs de Comarques (Roses; 1999) (Abstract).

6. Jimenez J, Garcia M, Martinez J, De la Fuente A, Zuloaga A. Tumores metastáticos en pene. Congreso Nacional de Urología (AEU; Málaga: 1993) (Abstract / P.14 - P41).

7. Lopez de Alda A, Rodriguez J, Garcia de la Peña E, Brito M Balauz A, Barat A. Metástasis peneana por carcinoma prostático. A propósito de un caso. Actas Urol Esp. 1990;14:163164.

8. Llopis B, Alonso M, Valls F, Moreno B, Boronat F, Gallego J, Iranzo S, Oliver F, Jiménez F. Tumores secundarios de pene. Actas Urol Esp. 1986;10:167-170
Tabla 9 (A y B)

Tumores Metastáticos Peneanos

Casuística estado español (60 casos: 1980 - 2005)

Procedimientos quirúrgicos (diagnóstico-terapéuticos) empleados como opción principal y/o primera

Exéresis biópsica lesional ..........................39 casos

Paaf y/o legrado cuerpos cavernosos ..............10 casos

Falectomía parcial ................................... casos

Falectomía total ....................................5 casos

Emasculación............................................ 1 caso

Total ...............................................60 casos

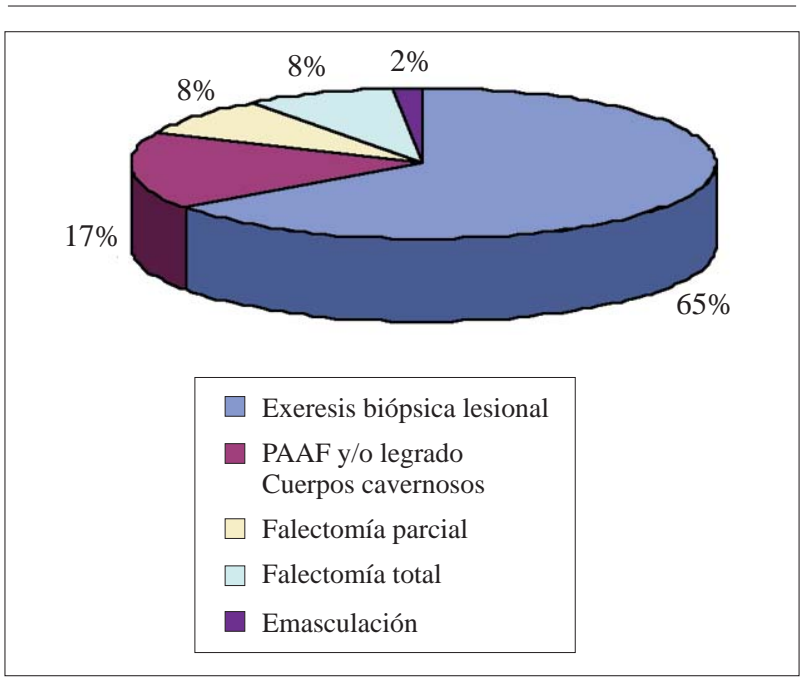

9. Ortiz R, Adriazola M, Blanco M, Garcia E, Tejeda E, Romero F. Lesiones cutáneas de pene. Metástasis de adenocarcinoma de próstata. Aportación de un caso. Actas Urol Esp. 1999;23:153-155.

10. Marchal C, Contreras J, Chicharro J, Diaz F, Funez R, Martin A, Burgos R. Enfermedad metastásica del pene. A propósito de tres casos. Actas Urol. Esp. 1993;17:461-463.

11. Morga J, Ferrero R, Guzman P, Navas J, Garcia J, Garcia F, Sempere A, Rico J, Tomas M, Fontana L. Priapismo metastásico. Presentación de cuatro nuevos casos y revisión de la literatura. Arch Esp Urol. 2000;53:447-452.

12. Muro I, Aspiazu P, Recarte J, Hernaez I: Carcinoma metastásico de pene: Evaluación clínica, tratamiento y revisión de la literatura. Arch Esp Urol. 1999;52:994.

13. Romero P, Amat M, Andrada E. Metástasis en glande de adenocarcinoma prostático. A propósito de un caso. Actas Urol Esp. 1991;15:284-287.

14. Salvador J, Orsola A, Xavier B, Sanchotene F, Algaba. Metástasis en el pene. Congreso Nacional de Urología (AEU; SITGES: 1994). (Abstract / P.80 - p.69).

15. Sanz E, Burgos F, Gomez I, Garcia R, Escudero A. Metástasis peneana de adenocarcinoma prostático. Arch Esp Urol. 2004;57:841-844.

Dr. C. Pellicé i Vilalta

Clínica de Nuestra Señora del Remedio

Escorial, 148

08024 Barcelona

E-mail:15256cpv@comb.es 